\title{
LATTICE SEPARATION AND MEASURES
}

\author{
JAMES PONNLEY
}

Received 26 July 2001

\begin{abstract}
The effects of lattice separation such as normality, almost normal, slightly normal on various lattice-derived measure are investigated and generalizations of earlier work on 0-1 valued measures are obtained.
\end{abstract}

2000 Mathematics Subject Classification: 28C15, 28A12.

1. Introduction. In an earlier paper [6], we considered a variety of special 0-1 valued measures and studied some associated outer measures and their measurable sets. A portion of this paper was then extended in [7, 8] to the more general case where the measures involved need not be just 0-1 valued. However, there were still sections of [6] that were not generalized, especially those related to separation; namely, where the lattice of subsets involved was slightly normal or almost normal. The extension of these results involves a number of different concepts that did not arise in the $0-1$ valued case, and the arguments involved are considerably different from the $0-1$ valued case.

We pursue these matters in Sections 3 and 4. In Section 5, we give some further related type theorems. Again, the major concern is how certain lattice separation properties affect various measures defined on the algebra generated by the lattice. In many cases regularity is implied, in other cases equality of certain associated outer measures is assured on various sets.

We begin in Section 2 with a brief review of some terminology and notation used throughout the paper. Also, a number of basic results are stated which are used throughout the paper. More specific facts are given in the sections to which they are most closely related.

2. Background and basic notation. We briefly review here some standard notation and terminology which are consistent with our previous usage in [6, 8]. The set $X$ denotes a nonempty arbitrary set, and $\mathscr{L}$ a lattice of subsets of $X$. All lattices considered throughout the paper will contain $\varnothing$ and $X$. The algebra $\mathscr{A}(\mathscr{L})$ denotes the algebra generated by $\mathscr{L}$, and $M(\mathscr{L})$ denotes those nontrivial, finite, nonnegative, and finitely additive measures on $\mathscr{A}(\mathscr{L})$. The set $M_{R}(\mathscr{L})$ denotes those elements of $\mu \in M(\mathscr{L})$ that are $\mathscr{L}$-regular. The set $M_{\sigma}(\mathscr{L})$ denotes those $\mu \in M(\mathscr{L})$ which are $\sigma$-smooth on $\mathscr{L}$; that is, if $L_{n}$ is monotonically decreasing to empty set, $\left(L_{n} \downarrow \varnothing\right), L_{n} \in \mathscr{L}$, then $\lim \mu\left(L_{n}\right)=0$. 
The set $M^{\sigma}(\mathscr{L})$ stands for those elements of $M(\mathscr{L})$ which are $\sigma$-smooth on $\mathscr{A}(\mathscr{L})$, and, consequently countably additive. The set $M_{R}^{\sigma}(\mathscr{L})$ stands for those elements $\mu \in M(\mathscr{L})$ that are common to both sets $M_{R}(\mathscr{L})$ and $M_{\sigma}(\mathscr{L})$, that is, $M_{R}^{\sigma}(\mathscr{L})=M_{R}(\mathscr{L}) \cap M_{\sigma}(\mathscr{L})$, and it is not difficult to see that if $\mu \in M_{R}^{\sigma}(\mathscr{L})$, then $\mu \in M^{\sigma}(\mathscr{L})$.

To a $\mu \in M(\mathscr{L})$, we associate a number of outer measures. Let $E \subset X$ and define

$$
\mu^{\prime}(E)=\inf \left\{\mu\left(L^{\prime}\right): E \subset L^{\prime}, L \in \mathscr{L}\right\}
$$

where $L^{\prime}=X-L$;

$$
\mu^{\prime \prime}(E)=\inf \left\{\sum_{i=1}^{\infty} \mu\left(L_{i}^{\prime}\right): E \subset \bigcup_{1}^{\infty} L_{i}^{\prime}, L_{i} \in \mathscr{L}\right\} .
$$

Similarly, we defined $\tilde{\mu}(E)$ and $\tilde{\tilde{\mu}}(E)$ where in the above definitions we replace the $L^{\prime}$ and $L_{i}^{\prime}$ by $L$ and $L_{i}$, respectively, where the $L, L_{i} \in \mathscr{L} ; \mu^{\prime}, \tilde{\mu}$ are finitely subadditive outer measures while $\mu^{\prime \prime}, \tilde{\tilde{\mu}}$ are countably subadditive outer measures.

In general, if $v_{1}, v_{2}$ are two set functions defined on a lattice $\mathscr{L}$, we write $v_{1} \leq v_{2}(\mathscr{L})$ if $v_{1}(L) \leq v_{2}(L)$, for all $L \in \mathscr{L}$.

We recall some simple relations involving these outer measures.

THEOREM 2.1. (a) $\mu^{\prime \prime} \leq \mu^{\prime}, \tilde{\tilde{\mu}} \leq \tilde{\mu}$.

(b) If $\mu \in M_{\sigma}(\mathscr{L})$, then $\mu^{\prime \prime}(X)=\mu(X)$ and $\mu \leq \mu^{\prime \prime}(\mathscr{L})$.

(c) If $\mu \in M_{\sigma}\left(\mathscr{L}^{\prime}\right)$, then $\tilde{\tilde{\mu}}(X)=\mu(X)$ and $\mu \leq \tilde{\tilde{\mu}}\left(\mathscr{L}^{\prime}\right)$. (See [2] for details).

Next, we recall that if $v$ is a regular countably subadditive outer measure, and if $E_{n}$ is monotonically increasing to $E,\left(E_{n} \uparrow E\right), E_{n} \subset X$, then

$$
v\left(\lim _{n \rightarrow \infty} E_{n}\right)=\lim _{n \rightarrow \infty} v\left(E_{n}\right) \quad(\text { see }[5]) .
$$

In this connection, we note that if $\mu \in M(\mathscr{L})$ and if $\mu^{\prime \prime}$ is a regular outer measure such that $\mu^{\prime \prime}(X)=\mu(X)$, then $\mu \in M_{\sigma}(\mathscr{L})$ (see [12]). A similar statement holds when $\tilde{\tilde{\mu}}$ is a regular outer measure.

We next recall that a lattice $\mathscr{L}$ is normal if whenever $A, B \in \mathscr{L}$ and $A \cap B=\varnothing$, there exist $C, D \in \mathscr{L}$ such that $A \subset C^{\prime}, B \subset D^{\prime}$, and $C^{\prime} \cap D^{\prime}=\varnothing$. If $\mathscr{L}_{1}$ and $\mathscr{L}_{2}$ are two lattices of subsets of $X$, then $\mathscr{L}_{1}$ is said to semiseparate $\mathscr{L}_{2}$ if whenever $A \in \mathscr{L}_{1}, B \in \mathscr{L}_{2}$, and $A \cap B=\varnothing$, there exists a $C \in \mathscr{L}_{1}$ with $B \subset C$ and $A \cap C=\varnothing$. The lattice $\mathscr{L}_{1}$, is said to separate $\mathscr{L}_{2}$ if for $A, B \in \mathscr{L}_{2}, A \cap B=\varnothing$, there exist $C, D \in \mathscr{L}_{1}$, such that $A \subset C, B \subset D$, and $C \cap D=\varnothing$. Finally, $\mathscr{L}_{1}$ coseparates $\mathscr{L}_{2}$ if, for $A, B \in \mathscr{L}_{2}, A \cap B=\varnothing$, there exist $C, D \in \mathscr{L}_{1}$ such that $A \subset C^{\prime}, B \subset D^{\prime}$, and $C^{\prime} \cap D^{\prime}=\varnothing$.

Detailed measure characterizations of these concepts can be found in $[1,6]$.

Finally, if $\mathscr{L}$ is a lattice of subsets of $X$, we denote by $\delta(\mathscr{L})$ the delta lattice generated by $\mathscr{L}$; that is, the smallest lattice containing $\mathscr{L}$ and closed under 
countable intersections. We also denote by $\mathscr{L}^{\prime}$ the set $\left\{L^{\prime}: L \in \mathscr{L}\right\}$, and if $v$ is an outer measure either finitely or countably subadditive, $\mathscr{S}_{v}$ designates the $v$-measurable sets.

3. The general case (a). In this section, we generalize a number of theorems established in [6] for the special case of 0-1 valued measures to the more general case. We denote for $\mu \in M(\mathscr{L})$, and

$$
E \subset X, \quad \mu_{i}(E)=\sup \{\mu(L): L \subset E, L \in \mathscr{L}\},
$$

where $\mu_{i}$ is an inner measure, and

$$
\mu_{i}(E)=\mu(X)-\mu^{\prime}\left(E^{\prime}\right)
$$

(for details on $\mu^{\prime}, \mu_{i}$, and related matters of measurability, see [2]).

Also, for $\mu \in M_{\sigma}\left(\mathscr{L}^{\prime}\right)$ and $E \subset X$,

$$
\mu_{k}(E)=\tilde{\tilde{\mu}}(X)-\tilde{\tilde{\mu}}\left(E^{\prime}\right)=\mu(X)-\tilde{\tilde{\mu}}\left(E^{\prime}\right)
$$

by Theorem 2.1(c). $\mu_{k}$ is not, in general, an inner measure; it is, if $\tilde{\tilde{\mu}}$ is submodular (see [4]).

THEOREM 3.1. Let $\mathscr{L}$ be a lattice of subsets of $X$, and let $\mu \leq \nu(\mathscr{L}), \mu(X)=$ $\nu(X)$, where $\mu \in M_{\sigma}\left(\mathscr{L}^{\prime}\right)$ and $v \in M_{R}(\mathscr{L})$. If $\tilde{\tilde{\mu}}$ is a regular outer measure and if $\delta\left(\mathscr{L}^{\prime}\right)$ separates $\mathscr{L}$, then

$$
v\left(L^{\prime}\right)=\sup \left\{\mu_{k}\left(\bigcup_{j=1}^{\infty} B_{j}\right): \bigcup B_{j} \subset L^{\prime}, B_{j} \in \mathscr{L}\right\}, \quad L \in \mathscr{L} .
$$

Proof. Since $\mu \leq v(\mathscr{L})$ and $\mu(X)=v(X), v \leq \mu\left(\mathscr{L}^{\prime}\right)$, so $v \in M_{\sigma}\left(\mathscr{L}^{\prime}\right)$, and, therefore, $\mu \leq \tilde{\tilde{\mu}}\left(\mathscr{L}^{\prime}\right)$ and $v \leq \tilde{\tilde{v}}\left(\mathscr{L}^{\prime}\right)$. Also, since $v \in M_{R}(\mathscr{L})$, there exists an $A \subset L^{\prime}, A \in \mathscr{L}$ such that $v\left(L^{\prime}\right)-v(A)<\epsilon$, where $\epsilon$ is an arbitrary positive number. Since $\delta\left(\mathscr{L}^{\prime}\right)$ separates $\mathscr{L}$, there exist $A_{i}, B_{j} \in \mathscr{L}$ such that

$$
A \subset \bigcap A_{i}^{\prime} \subset \bigcup B_{j} \subset L^{\prime}
$$

and where we may assume that the $A_{i}^{\prime}$ is monotonically decreasing to $A,\left(A_{i}^{\prime} \downarrow A\right)$ and the $B_{j}$ is monotonically increasing to $L^{\prime},\left(B_{j} \uparrow L^{\prime}\right)$.

Clearly, $v(A) \leq v_{i}\left(\cap A_{i}^{\prime}\right)$. Now,

$$
\begin{aligned}
\tilde{\mu}\left(\bigcup A_{i}\right) & =\lim \tilde{\tilde{\mu}}\left(A_{i}\right) \quad \text { (since } \tilde{\mu} \text { is regular) } \\
& \leq \lim v^{\prime}\left(A_{i}\right) \leq v^{\prime}\left(\bigcup A_{i}\right) .
\end{aligned}
$$

Hence,

$$
\mu_{k}\left(\bigcap A_{i}^{\prime}\right)=\mu(X)-\tilde{\tilde{\mu}}\left(\bigcup A_{i}\right) \geq v(X)-v^{\prime}\left(\bigcup A_{i}\right)=v_{i}\left(\bigcap A_{i}^{\prime}\right) .
$$


Therefore,

$$
\begin{aligned}
v(A) & \leq v_{i}\left(\bigcap A_{i}^{\prime}\right) \leq \mu_{k}\left(\bigcap A_{i}^{\prime}\right) \leq \mu_{k}\left(\bigcup B_{j}\right) \\
& \leq \tilde{\tilde{\mu}}\left(\bigcup B_{j}\right)=\lim \tilde{\tilde{\mu}}\left(B_{j}\right) \leq \lim \mu\left(B_{j}\right) \\
& \leq \lim v\left(B_{j}\right) \leq v^{\prime}\left(\bigcup B_{j}\right) \leq v\left(L^{\prime}\right) .
\end{aligned}
$$

From which the result follows immediately.

COROLLARY 3.2. Let $\mathscr{L}$ be a lattice of subsets of $X$, and let $\mu \in M_{\sigma}\left(\mathscr{L}^{\prime}\right)$ such that $\tilde{\tilde{\mu}}$ is a regular outer measure. If $v \in M_{R}(\mathscr{L})$ is such that $\mu \leq \nu(\mathscr{L}), \mu(X)=$ $v(X)$, and if $\delta\left(\mathscr{L}^{\prime}\right)$ separates $\mathscr{L}$, then $v$ is unique.

Proof. The proof that such a $v \in M_{R}(\mathscr{L})$ exists is well known (see $\left.[3,10]\right)$. The uniqueness follows immediately from the theorem, since if $v_{1}, v_{2} \in M_{R}(\mathscr{L})$ both satisfy the conditions, then $v_{1}=v_{2}\left(\mathscr{L}^{\prime}\right)$, and, therefore, $v_{1}=v_{2}$. We denote by $I(\mathscr{L})$ the 0 -1 valued measures of $M(\mathscr{L})$, and similarly, for the other subsets of $M(\mathscr{L})$; for example, $I_{\sigma}(\mathscr{L})$ denotes those elements of $I(\mathscr{L})$ that are $\sigma$-smooth on $\mathscr{L}$. We note that any 0 -1 valued outer measure is trivially regular. Also recall the following definition.

Definition 3.3. The lattice $\mathscr{L}$ is slightly normal if for $\mu \in I_{\sigma}\left(\mathscr{L}^{\prime}\right)$ and $\mu \leq$ $v_{1}(\mathscr{L}), \mu \leq v_{2}(\mathscr{L})$, where $v_{1}, v_{2} \in I_{R}(\mathscr{L})$ implies $v_{1}=v_{2}$.

Hence, as a special case of Corollary 3.2, we get the following result of [6].

COROLLARY 3.4. If $\delta\left(\mathscr{L}^{\prime}\right)$ separates $\mathscr{L}$, then the lattice $\mathscr{L}$ is slightly normal.

We note that if $\mathscr{L}^{\prime}$ itself separates $\mathscr{L}$, that is, if $\mathscr{L}$ is normal, then the set inclusions in the proof of Theorem 3.1 become simply $A \subset A_{1}^{\prime} \subset B \subset L^{\prime}$, and in this case, it is easy to see that

$$
v(A) \leq v\left(A_{1}^{\prime}\right) \leq \mu\left(A_{1}^{\prime}\right) \leq \mu(B) \leq v(B) \leq v\left(L^{\prime}\right)
$$

without any need for $\mu$ to belong to $M_{\sigma}\left(\mathscr{L}^{\prime}\right)$, or for $\tilde{\tilde{\mu}}$ to be regular, (3.9) of course implies that $v=\mu_{i}\left(\mathscr{L}^{\prime}\right)$, or, equivalently, $v=\mu^{\prime}(\mathscr{L})$. Thus, we have the following corollary.

COROLlaRY 3.5. Let $\mathscr{L}$ be a lattice of subsets of $X$, and let $\mu \leq \nu(\mathscr{L}), \mu(X)=$ $v(X)$ where $\mu \in M(\mathscr{L})$ and $v \in M_{R}(\mathscr{L})$. If $\mathscr{L}$ is normal, then

(a) $v=\mu^{\prime}(\mathscr{L}), v=\mu_{i}\left(\mathscr{L}^{\prime}\right)$;

(b) $v$ is unique.

We can use the result to obtain a simple proof of the following corollary.

COROLlaRY 3.6. Let $\mathscr{L}$ be a lattice of subsets of $X$, and let $\mu \leq \nu(\mathscr{L}), \mu(X)=$ $v(X)$, where $\mu \in M_{\sigma}(\mathscr{L}), v \in M_{R}(\mathscr{L})$. If $\mathscr{L}$ is normal, then $v \in M_{\sigma}\left(\mathscr{L}^{\prime}\right)$. 
Proof. Let $A_{n}^{\prime} \downarrow \varnothing, A_{n} \in \mathscr{L}$, then by Corollary 3.5(a), there exists $B_{n} \subset A_{n}^{\prime}$, $B_{n} \in \mathscr{L}$, which we may assume $\downarrow$ such that

$$
v\left(A_{n}^{\prime}\right)<\mu\left(B_{n}\right)+\epsilon,
$$

where $\epsilon>0$ is arbitrary.

Since $\cap B_{n}=\varnothing, \mu\left(B_{n}\right) \rightarrow 0$; whence $v \in M_{\sigma}\left(\mathscr{L}^{\prime}\right)$.

The last two corollaries are known, but shown in a different manner (see [2]).

4. The general case (b). In this section, we extend the results of [6] pertaining to almost normal lattices.

Recall the following definition.

DEFINITION 4.1. The lattice $\mathscr{L}$ is almost normal if, for $A, B \in \mathscr{L}$ and $A \cap B=$ $\varnothing$, there exist $A_{i}^{\prime} \uparrow, A_{i} \in \mathscr{L}$ such that $A \subset \cup_{1}^{\infty} A_{i}^{\prime}$, and there exist $B_{i} \in \mathscr{L}$ with $A_{i}^{\prime} \subset B_{i}$, for all $i$ and $B_{i} \cap B=\varnothing$, for all $i$.

It is not difficult to show that if $\mathscr{L}$ is a delta lattice, and if $\mathscr{L}$ is almost normal, then $\mathscr{L}$ is normal.

We now have the following theorem.

THEOREM 4.2. Let $\mathscr{L}$ be a lattice of subsets of $X$ which is almost normal. Suppose $\mu \leq v(\mathscr{L}), \mu(X)=v(X)$, where $\mu \in M(\mathscr{L})$ and $v \in M_{R}^{\sigma}(\mathscr{L})$ and where $\mu^{\prime \prime}$ is a regular outer measure. Then $\mu^{\prime \prime}=\nu^{\prime \prime}(\mathscr{L})$.

Proof. We note that since $v \in M_{R}^{\sigma}(\mathscr{L}), \mu \in M_{\sigma}(\mathscr{L})$. Also since $\mu \leq v(\mathscr{L})$ and $\mu(X)=v(X), v^{\prime \prime} \leq \mu^{\prime \prime}$ and in particular $\nu^{\prime \prime} \leq \mu^{\prime \prime}(\mathscr{L})$. Suppose that there exists an $A \in \mathscr{L}$ such that $v^{\prime \prime}(A)<\mu^{\prime \prime}(A)$. We note that $v=v^{\prime \prime}=v^{\prime}(\mathscr{L})$ since $v \in M_{R}^{\sigma}(\mathscr{L})$.

Hence,

$$
v(A) \leq v^{\prime \prime}(A)<\mu^{\prime \prime}(A) .
$$

Then there exists a $B \in \mathscr{L}$ such that $B^{\prime} \supset A$, and

$$
v(A) \leq v\left(B^{\prime}\right)<\mu^{\prime \prime}(A) .
$$

Since $A \cap B=\varnothing$, there exist $A_{i}^{\prime} \uparrow, A_{i} \in \mathscr{L}$, such that $A \subset \cup A_{i}^{\prime}$, and there exist $B_{i} \in \mathscr{L}$ with $A_{i}^{\prime} \subset B_{i}, B_{i} \cap B=\varnothing$, for all $i$.

Hence,

$$
\begin{aligned}
\mu^{\prime \prime}(A) & \leq \mu^{\prime \prime}\left(\bigcup A_{i}^{\prime}\right)=\lim \mu^{\prime \prime}\left(A_{i}^{\prime}\right) \leq \lim \mu\left(A_{i}^{\prime}\right) \\
& \leq \varlimsup \lim \mu\left(B_{i}\right) \leq \varlimsup \lim v\left(B_{i}\right) \leq v^{\prime}\left(\bigcup B_{i}\right) \\
& \leq v\left(B^{\prime}\right)<\mu^{\prime \prime}(A),
\end{aligned}
$$

a contradiction. Hence $v^{\prime \prime}=\mu^{\prime \prime}(\mathscr{L})$. 
We note that Theorem 4.2 can be generalized. For this purpose, recall the following definition (see [9]).

DEFINITION 4.3. Let $\mu \in M_{\sigma}(\mathscr{L}), \mu$ is called vaguely regular if $\mu\left(A^{\prime}\right)=$ $\sup \left\{\mu^{\prime \prime}(B): B \subset A^{\prime}, B \in \mathscr{L}\right\}$ for $A \in \mathscr{L}$.

The set of vaguely regular measures is denoted by $M_{v}(\mathscr{L})$. For $\mu \in M_{\sigma}(\mathscr{L})$, $E \subset X$, let

$$
\mu_{j}(E)=\mu^{\prime \prime}(X)-\mu^{\prime \prime}\left(E^{\prime}\right)=\mu(X)-\mu^{\prime \prime}\left(E^{\prime}\right)
$$

Then we have $\mu \in M_{v}(\mathscr{L})$ if and only if

$$
\mu(A)=\inf \left\{\mu_{j}\left(B^{\prime}\right): A \subset B^{\prime}, B \in \mathscr{L}\right\}, \quad \text { for } A \in \mathscr{L} .
$$

It is, of course, clear that $M_{R}^{\sigma}(\mathscr{L}) \subset M_{v}(\mathscr{L})$, and we can now establish the following generalization of Theorem 4.2.

THEOREM 4.4. Let $\mathscr{L}$ be a lattice of subsets of $X$ which is almost normal. Suppose that $\mu \leq v(\mathscr{L}), \mu(X)=v(X)$ where $\mu \in M(\mathscr{L})$ and $v \in M_{v}(\mathscr{L})$ and where $\mu^{\prime \prime}$ is a regular outer measure. Then $\mu^{\prime \prime}=\nu^{\prime \prime}(\mathscr{L})$.

Proof. As in the proof of Theorem 4.2, we suppose that there exists an $A \in \mathscr{L}$ such that $\nu^{\prime \prime}(A)<\mu^{\prime \prime}(A)$ and will arrive at a contradiction. There exists a $B \in \mathscr{L}$, such that $B^{\prime} \supset A$ and

$$
v(A) \leq v_{j}\left(B^{\prime}\right)<\mu^{\prime \prime}(A) .
$$

Then, there exists $A_{i}^{\prime} \uparrow, A_{i} \in \mathscr{L}$, such that $A \subset \cup A_{i}^{\prime}$, and there exists $B_{i} \in \mathscr{L}$ with $A_{i}^{\prime} \subset B_{i}, B_{i} \cap B=\varnothing$, for all $i$. Finally, we note that since $v \in M_{v}(\mathscr{L}), v^{\prime \prime}=v^{\prime}=$ $v\left(\mathscr{L}^{\prime}\right)$. Thus, we have

$$
\begin{aligned}
& \mu^{\prime \prime}(A) \leq \mu^{\prime \prime}\left(\bigcup A_{i}^{\prime}\right)=\lim \mu^{\prime \prime}\left(A_{i}^{\prime}\right) \leq \lim \mu\left(A_{i}^{\prime}\right)
\end{aligned}
$$

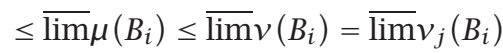

$$
\begin{aligned}
& \leq v_{j}\left(\bigcup B_{i}\right) \leq v_{j}\left(B^{\prime}\right)<\mu^{\prime \prime}(A) \text {, }
\end{aligned}
$$

a contradiction, and we are done.

Again, recalling that any 0-1 valued outer measure is regular, we get as special cases of the preceding theorems, the following result of [6].

COROLLARY 4.5. Let $\mathscr{L}$ be a lattice of subsets of $X$ which is almost normal. If $\mu \leq \nu(\mathscr{L})$ where $\mu \in I(\mathscr{L})$, and where $\mu \in I_{R}^{\sigma}(\mathscr{L})$ or, more generally, $\mu \in I_{v}(\mathscr{L})$, then $v^{\prime \prime}=\mu^{\prime \prime}(\mathscr{L})$.

Finally, we note the following corollary to Theorem 4.2. 
COROLLARY 4.6. Let $\mathscr{L}$ be a lattice of subsets of $X$ which is almost normal. If $\mu \leq v_{1}(\mathscr{L}), \mu \leq v_{2}(\mathscr{L})$ where $\mu(X)=v_{1}(X)=v_{2}(X), \mu \in M(\mathscr{L}), v_{1}, v_{2} \in M_{R}^{\sigma}(\mathscr{L})$ and if $\mu^{\prime \prime}$ is a regular outer measure, then $\nu_{1}=\nu_{2}$.

Proof. By Theorem 4.2,

$$
v_{1}=v_{1}^{\prime \prime}=\mu^{\prime \prime}=v_{2}^{\prime \prime}=v_{2}(\mathscr{L}) .
$$

Hence, $v_{1}=v_{2}$.

5. Further extensions. We begin by recalling the following definition.

Definition 5.1. Let $\mu \in M(\mathscr{L})$. Then $\mu \in M_{w}(\mathscr{L})$ (the weakly regular measures) if, for $L \in \mathscr{L}$,

$$
\mu\left(L^{\prime}\right)=\sup \left\{\mu^{\prime}(\tilde{L}): \tilde{L} \subset L^{\prime}, \tilde{L} \in \mathscr{L}\right\} .
$$

Clearly, $M_{R}(\mathscr{L}) \subset M_{w}(\mathscr{L})$, and $M_{v}(\mathscr{L}) \subset M_{w}(\mathscr{L})$.

The following result in [6] has been generalized in [12].

THEOREM 5.2. If $\mathscr{L}$ is a lattice of subsets of $X$ such that $\delta\left(\mathscr{L}^{\prime}\right)$ separates $\mathscr{L}$, then $\mu \in I_{\sigma}\left(\mathscr{L}^{\prime}\right) \cap I_{w}(\mathscr{L})$ implies that $\mu \in I_{R}(\mathscr{L})$.

For completeness we state and prove the generalization, correcting a reference which appears in [11].

THEOREM 5.3. If $\mathscr{L}$ is a lattice of subsets of $X$ such that $\delta\left(\mathscr{L}^{\prime}\right)$ separates $\mathscr{L}$, then $\mu \in M_{\sigma}\left(\mathscr{L}^{\prime}\right) \cap M_{w}(\mathscr{L})$ implies that $\mu \in M_{R}(\mathscr{L})$.

Proof. We recall (see [2]) that

$$
\mathscr{Y}_{\mu^{\prime}} \cap \mathscr{L}=\left\{L \in \mathscr{L}: \mu^{\prime}(L)=\mu(L)\right\} .
$$

Hence, if we can show that $\mathscr{L} \subset \mathscr{Y}_{\mu^{\prime}}$, then $\mu^{\prime}=\mu(\mathscr{L})$, and $\mu \in M_{R}(\mathscr{L})$. To this end, let $\epsilon>0$ and $A \in \mathscr{L}$. Since $\mu \in M_{w}(\mathscr{L})$, there exists a $B \in \mathscr{L}$ such that $B \subset A^{\prime}$, and $\mu\left(A^{\prime}\right)-\epsilon / 2<\mu^{\prime}(B) \leq \mu\left(A^{\prime}\right)$.

Now, there exists $\cap_{1}^{\infty} A_{n}^{\prime}, \cap_{1}^{\infty} B_{n}^{\prime} \in \delta(\mathscr{L})$ with $A_{n}^{\prime} \downarrow, B_{n}^{\prime} \downarrow$, and $A \subset \cap_{1}^{\infty} A_{n}^{\prime}, B \subset$ $\cap_{1}^{\infty} B_{n}^{\prime}$, and $\cap_{1}^{\infty}\left(A_{n}^{\prime} \cap B_{n}^{\prime}\right)=\varnothing$.

Now $\mu \in M_{\sigma}\left(\mathscr{L}^{\prime}\right)$; hence $\mu\left(A_{n}^{\prime} \cap B_{n}^{\prime}\right) \rightarrow 0$, so $\mu\left(A_{n}^{\prime} \cap B_{n}^{\prime}\right)<\epsilon / 2$ for $n \geq N$. But

$$
\mu\left(A_{n}^{\prime} \cup B_{n}^{\prime}\right)=\mu\left(A_{n}^{\prime}\right)+\mu\left(B_{n}^{\prime}\right)-\mu\left(A_{n}^{\prime} \cap B_{n}^{\prime}\right) \geq \mu\left(A_{n}^{\prime}\right)+\mu\left(B_{n}^{\prime}\right)-\frac{\epsilon}{2} .
$$

Hence,

$$
\mu\left(A_{n}^{\prime} \cup B_{n}^{\prime}\right) \geq \mu^{\prime}(A)+\mu^{\prime}(B)-\frac{\epsilon}{2}
$$

Then

$$
\mu\left(A_{n}^{\prime} \cup B_{n}^{\prime}\right) \geq \mu^{\prime}(A)+\mu\left(A^{\prime}\right)-\epsilon
$$


or

$$
\mu\left(A_{n}^{\prime} \cup B_{n}^{\prime}\right) \geq \mu^{\prime}(A)+\mu^{\prime}\left(A^{\prime}\right)-\epsilon
$$

and therefore,

$$
\mu(X)=\mu^{\prime}(X) \geq \mu^{\prime}(A)+\mu^{\prime}\left(A^{\prime}\right)
$$

This implies (see [2]) that $A \in \mathscr{Y}_{\mu^{\prime}}$, and, since $A \in \mathscr{L}$ is arbitrary, this completes the proof.

A related result is the following.

THEOREM 5.4. Let $\mathscr{L}$ be a lattice of subsets of $X$ and let $\mu \in M_{w}(\mathscr{L}) \cap M_{\sigma}(\mathscr{L})$. If $\mu_{i}\left(L^{\prime}\right)=\sup \left\{\mu^{\prime \prime}(\tilde{L}): \tilde{L} \subset L^{\prime}, \tilde{L} \in \mathscr{L}\right\}$, for $L \in \mathscr{L}$, and if $\mathscr{L}$ semiseparates $\delta(\mathscr{L})$, then $\mu \in M_{R}^{\sigma}(\mathscr{L})$.

Proof. For $L \in \mathscr{L}$, we have

$$
\mu_{i}\left(L^{\prime}\right)=\mu_{j}\left(L^{\prime}\right)=\sup \left\{\mu^{\prime \prime}(\tilde{L}): \tilde{L} \subset L^{\prime}, \tilde{L} \in \mathscr{L}\right\}
$$

(see [7] for details). Hence, $\mu_{i}=\mu_{j}\left(\mathscr{L}^{\prime}\right)$ and, therefore,

$$
\mu^{\prime}=\mu^{\prime \prime}(\mathscr{L})
$$

Thus,

$$
\mu_{i}\left(L^{\prime}\right)=\sup \left\{\mu^{\prime}(\tilde{L}): \tilde{L} \subset L^{\prime}, \tilde{L} \in \mathscr{L}\right\}=\mu\left(L^{\prime}\right) \quad \text { since } \mu \in M_{w}(\mathscr{L}) .
$$

Hence, $\mu_{i}=\mu\left(\mathscr{L}^{\prime}\right)$, and this implies that $\mu \in M_{R}(\mathscr{L})$, so $\mu \in M_{R}(\mathscr{L}) \cap M_{\sigma}(\mathscr{L})=$ $M_{R}^{\sigma}(\mathscr{L})$.

In a slightly different direction, we give one more result which has significant applications.

THEOREM 5.5. Let $\mathscr{L}$ be a lattice of subsets of $X$, and let $\mu \leq v(\mathscr{L})$ where $\mu \in$ $M(\mathscr{L}), v \in M_{\sigma}(\mathscr{L})$, and $\mu(X)=v(X)$. If $\mathscr{L}$ is normal and if $\mu^{\prime \prime}\left(L^{\prime}\right)=\sup \left\{\mu^{\prime \prime}(A)\right.$ : $\left.A \subset L^{\prime}, A \in \mathscr{L}\right\}$, for $L \in \mathscr{L}$; then $\mu^{\prime \prime}=v^{\prime \prime}\left(\mathscr{L}^{\prime}\right)$.

Proof. Clearly, $\mu \in M_{\sigma}(\mathscr{L})$ and $\nu^{\prime \prime} \leq \mu^{\prime \prime}$. Suppose that there exists an $L^{\prime}, L \in \mathscr{L}$, such that $v^{\prime \prime}\left(L^{\prime}\right)<\mu^{\prime \prime}\left(L^{\prime}\right)$. By hypothesis, there then exists an $A \subset L^{\prime}$, $A \in \mathscr{L}$ such that $\nu^{\prime \prime}\left(L^{\prime}\right)<\mu^{\prime \prime}(A)$. Now, there exists $B, C \in \mathscr{L}$ such that $A \subset B^{\prime} \subset$ $C \subset L^{\prime}$. Then,

$$
\begin{aligned}
v^{\prime \prime}\left(L^{\prime}\right) & <\mu^{\prime \prime}(A) \leq \mu^{\prime \prime}\left(B^{\prime}\right) \leq \mu\left(B^{\prime}\right) \leq \mu(C) \\
& \leq v(C) \leq v^{\prime \prime}(C) \leq v^{\prime \prime}\left(L^{\prime}\right)
\end{aligned}
$$

a contradiction, so $v^{\prime \prime}=\mu^{\prime \prime}\left(\mathscr{L}^{\prime}\right)$. 
The result as mentioned has many applications; in particular, we note that in the case of a delta lattice and a measure $v \in M_{\sigma}(\mathscr{L}), v^{\prime \prime}$ will be submodular if it is submodular on $\mathscr{L}^{\prime}$. Theorem 5.5 assures us, under the stated hypothesis, that $\mu^{\prime \prime}$ will be submodular if and only if $\nu^{\prime \prime}$ is submodular. These facts are useful since submodularity of a $v \in M_{\sigma}(\mathscr{L})$ assures us that the set $\{E \subset X$ : $\left.\nu^{\prime \prime}(E)=\nu_{j}(E)\right\}$ is a $\sigma$-algebra, and that $\nu^{\prime \prime}$ restricted to this set is a countably additive measure (see [4]). We will not pursue these matters here.

\section{REFERENCES}

[1] P. M. Grassi, Measure characterizations and properties of normal and regular lattices, Int. J. Math. Math. Sci. 14 (1991), no. 2, 385-392.

[2] __ Outer measures and associated lattice properties, Int. J. Math. Math. Sci. 16 (1993), no. 4, 687-694.

[3] J. Kindler, Supermodular and tight set functions, Math. Nachr. 134 (1987), 131147.

[4] J. E. Knight, On finitely subadditive outer measures, J. Math. Sci. (Calcutta) 7 (1996), no. 2, 91-102.

[5] M. E. Munroe, Introduction to Measure and Integration, Addison-Wesley, Massachusetts, 1953.

[6] J. Ponnley, Outer measures, measurability, and lattice regular measures, Int. J. Math. Math. Sci. 19 (1996), no. 2, 343-350.

[7] __ On lattice associated outer measures and applications to smoothness conditions, J. Math. Sci. (Calcutta) 9 (1998), no. 1, 57-69.

[8]___ Lattice related outer measure inequalities and their applications, J. Math. Sci. (Calcutta) 10 (1999), no. 1, 21-32.

[9] D. Siegel, Outer measures and weak regularity of measures, Int. J. Math. Math. Sci. 18 (1995), no. 1, 49-58.

[10] M. Szeto, On maximal measures with respect to a lattice, Measure Theory and its Applications, 1980 Conference Northern Illinois Univ., 1981, pp. 277-282.

[11] C. Traina, Outer measures associated with lattice measures and their application, Int. J. Math. Math. Sci. 18 (1995), no. 4, 725-734.

[12] Smoothness conditions on measures using Wallman spaces, Int. J. Math. Math. Sci. 22 (1999), no. 4, 713-726.

James Ponnley: Department of Mathematical Sciences, Clark Atlanta University, Atlanta, GA 30314-4385, USA 


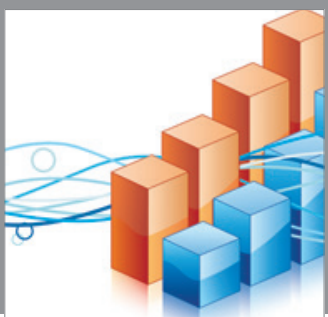

Advances in

Operations Research

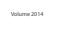

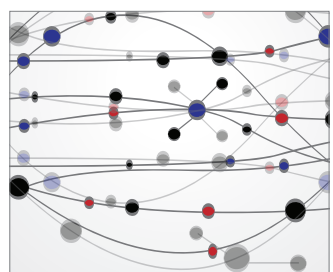

\section{The Scientific} World Journal
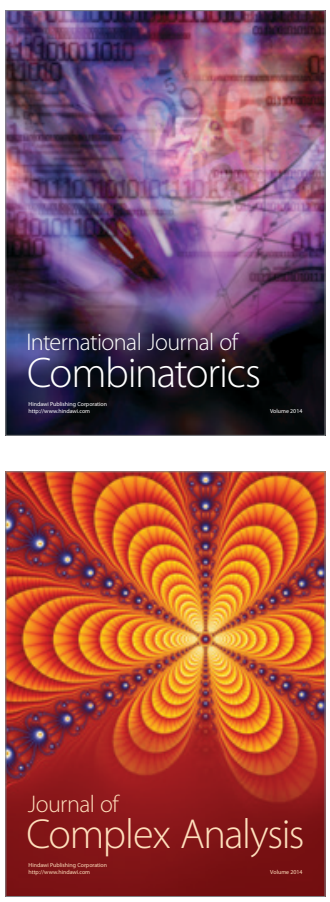

International Journal of

Mathematics and

Mathematical

Sciences
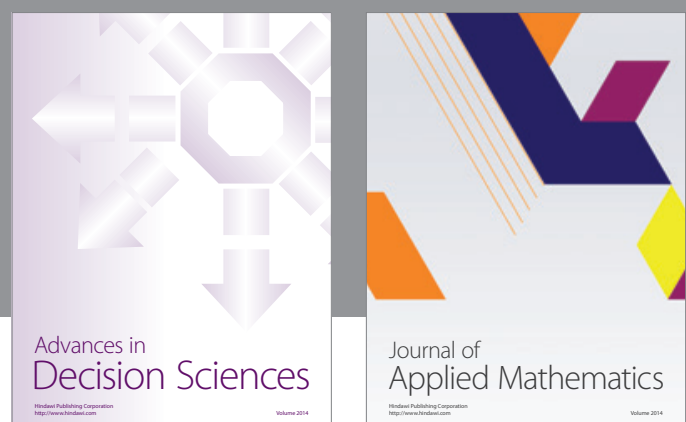

Journal of

Applied Mathematics
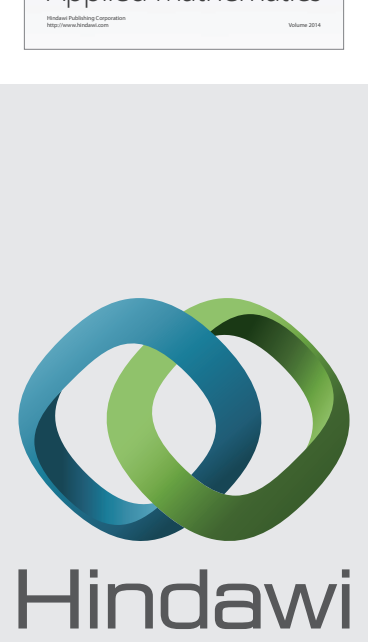

Submit your manuscripts at http://www.hindawi.com
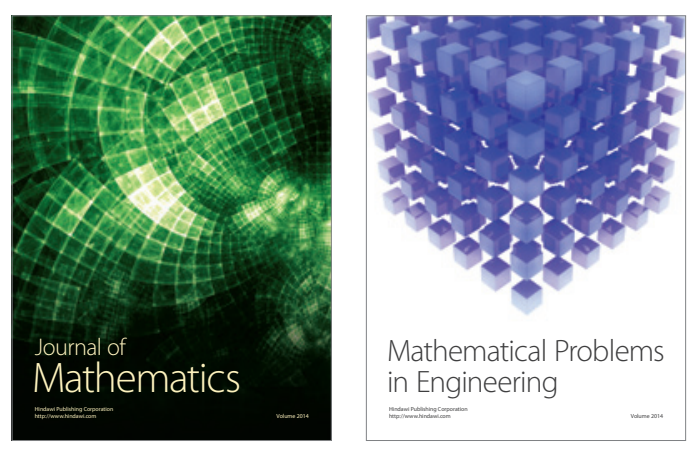

Mathematical Problems in Engineering
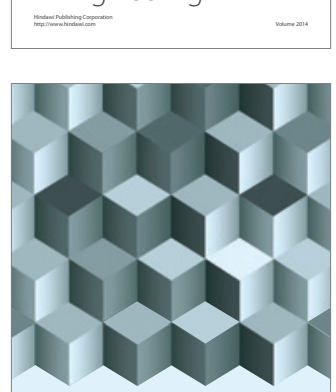

Journal of

Function Spaces
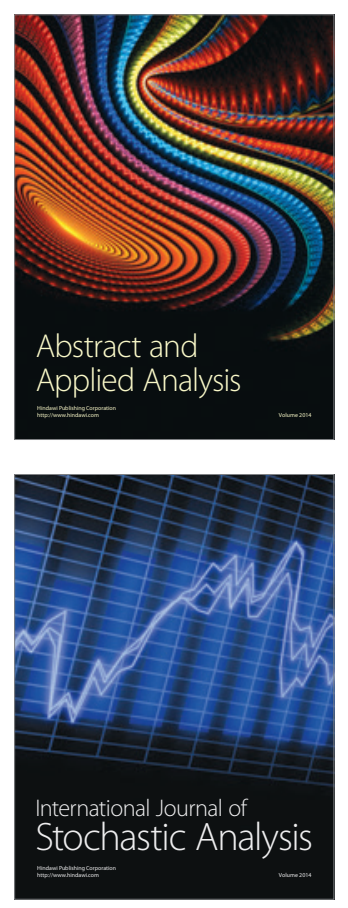

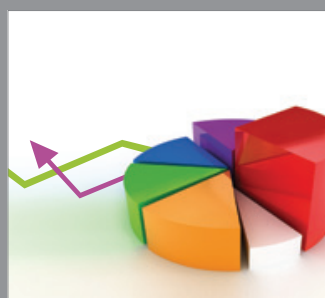

ournal of

Probability and Statistics

Promensencen
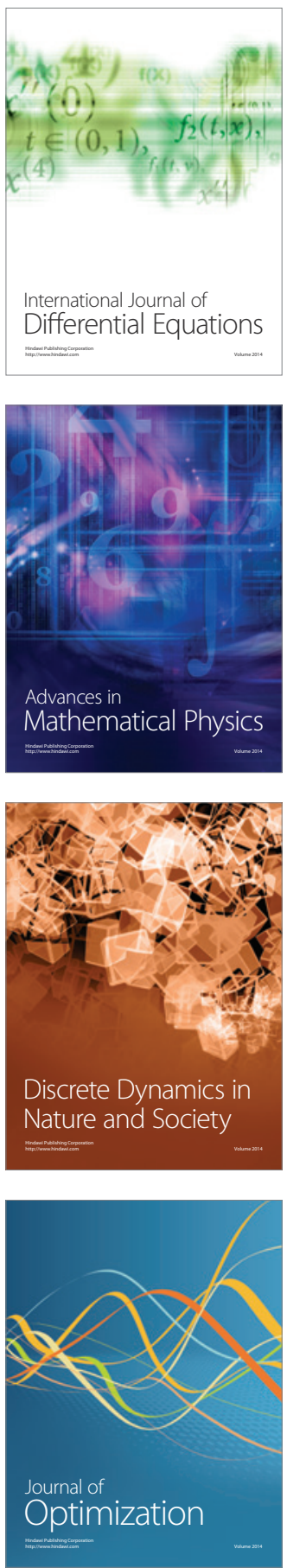